Here we report the detailed crystal structure of the $\mathrm{C} 2$ form obtained for the complexes of $\alpha$-cyclodextrin with decanol, undecanol and lauric acid. This crystal form is fully hydrated, with 25 water molecules at least for one dimer of $\alpha$-cyclodextrin in the asymmetric unit. Water channels lie between the columns. The lipid molecule has an all-trans elongated conformation in the tubular cavity formed by the $\alpha$-cyclodextrin dimer. The columnar structure is stabilized by hydrogen bonds but the interactions between the columns are different from those previously observed. Investigation of the hydrogen bonds is essential to understand how the water molecules influence the crystal packing and how the same complexes can crystallize in the $\mathrm{C} 2$ form as well as in the triclinic or hexagonal forms. X-ray diffraction measurements were made on the W32 beamline at LURE and on a Bruker SMART CCD area detector diffractometer at low temperature $(100 \mathrm{~K})$ in the "Ecole Centrale Paris". As these complexes yield suitable single crystals, neutron diffraction data were collected at $15 \mathrm{~K}$ on the new D19 instrument at ILL.

[1] Noltemeyer M., Saenger W., J. Am. Chem. Soc., 1980, 102(8), 2710-2722.

[2] Gallois-Montbrun D., Lesieur S., Ollivon M., Prangé T., Durand D., Le Bas G., Proceeding of the 12th International Cyclodextrin Symposium, Montpellier, France, May 16-19, 2004 [3] Gallois-Montbrun D., Lesieur S., Prangé T., Durand D., Ollivon M., Le Bas G., Acta Cryst., 2005, A61, C288.

\section{MS19 O4}

Cooperation of Tandem Hotdog Domains in Acyl-CoA

Thioesterase 7 Bostjan Kobe, Jade K. Forwood, Anil S. Thakur, Gregor Guncar, Mary Marfori, Dmitri Mouradov, Stuart Kellie, David A. Hume, Thomas Huber, Jennifer L. Martin, School of Molecular and Microbial Sciences and Institute for Molecular Bioscience, University of Queensland, Brisbane, Australia

E-mail: b.kobe@uq.edu.au

\section{Keywords: enzyme, thioesterase, hotdog domain}

We present a comprehensive structural and functional characterization of the enzyme acyl-CoA thioesterase 7 (Acot7). Acots catalyze the hydrolysis of fatty acyl-CoA to free fatty acid and coenzyme A, and thereby regulate lipid metabolism and cellular signalling. While prokaryotic homologues possess a single thioesterase domain, mammalian Acot7 contains a pair of domains in tandem. We determined the crystal structures of both the $\mathrm{N}$ - and C-terminal domains (1.8 and $2.5 \AA$ resolution, respectively) of the mouse enzyme, and inferred the structure of the full-length enzyme using a combination of chemical crosslinking, mass spectrometry, and molecular modelling. The novel quaternary arrangement features a trimer of hotdog fold dimers. We show that both domains of Acot7 are required for activity, that only one of two possible active sites in the dimer is functional, and identify the catalytic residues through site-directed mutagenesis. We also designed an enzyme with higher activity than wild-type Acot7 by mutating the residues in the nonfunctional active site. We demonstrate the highest enzyme activity against arachidonoyl-CoA (a precursor of eicosanoids), suggesting a role in inflammatory processes. Together, our results provide a foundation to relate the molecular and cellular functions of Acot7 in mammalian tissues.

\section{MS19 O5}

Crystal Engineering of Propargylic Alcohols-New Developments and Applications Marilise A. Hyacinth, ${ }^{a}$ Ashley R. Borges, ${ }^{\mathrm{b}}$ Michelle Lum, $^{\mathrm{b}}$ Maksymilian Chruszcz, ${ }^{\mathrm{c}}$ Kevin L. Caran, ${ }^{\mathrm{b}}$ Lin $\mathrm{Pu}^{\mathrm{a}}{ }^{\mathrm{a}}$ Michal Sabat ${ }^{\mathrm{a}}$ a Department of Chemistry, University of Virginia, Charlottesville,VA 22904, U.S.A. ${ }^{\mathrm{b}}$ Department of Chemistry, James Madison University, Harrisonburg, VA 22807, U.S.A. ${ }^{\mathrm{c} D e p a r t m e n t ~ o f ~ M o l e c u l a r ~ P h y s i o l o g y ~ a n d ~}$ Biological Physics, University of Virginia, Charlottesville, VA 22908, U.S.A. E-mail: mh5ua@,virginia.edu

Keywords: supramolecular chemistry, crystal engineering, cooperative phenomena

Propargylic alcohols, especially their diaryl-substituted chiral derivatives, possess functional groups that make these alcohols well-suited for the formation of supramolecular assemblies. Of special interest are cyclic hexameric systems occurring as a result of the cooperation between three major intermolecular forces: O-H...O hydrogen bonding, C-H...F-C hydrogen bonding involving organic fluorine atoms, and $\pi-\pi$

stacking interactions between the pentafluorophenyl and phenyl rings [1]. These hexagonal assemblies feature channels and cavities that can be used in several applications. Structural chemistry and possible applications of propargylic alcohols in gelation and as gas storage containers will be discussed.

[1]Hyacinth M., Chruszcz M., Lee K.S., Sabat M., Gao G., Pu, L. Angew.Chem. Int .Ed. 2006, 45. 5538 . 